Reaam A. Fadhil BDS, (Master student)

Saher S.Gasgoos BDS, MSc. (Asst. Prof.)

\section{Effect of Different Mouthwashes and Text Messages on Plaque Accumulation for Den- tal Students}

\author{
Ministry of Health-Nineveh Health Directorate
}

Depart. of Pedodontic, Ortho. and Preventive Dentistry College of Dentistry, University of Mosul

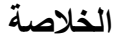

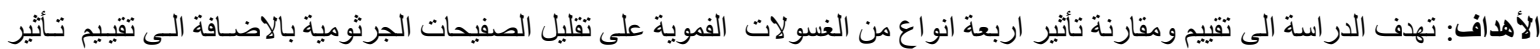

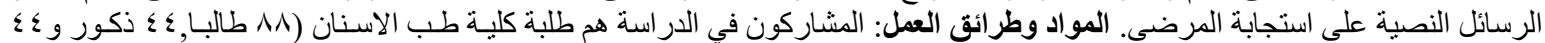

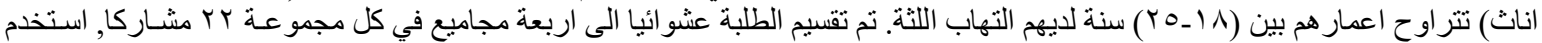

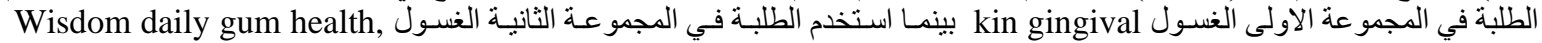

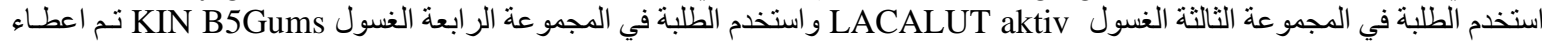

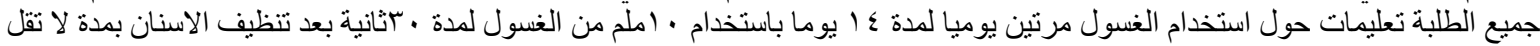

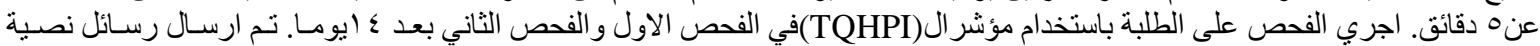

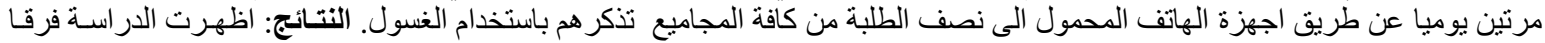

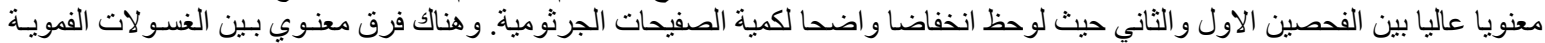

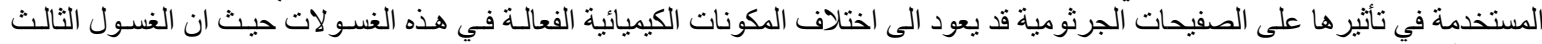

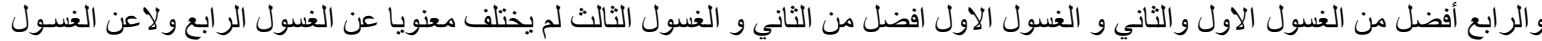

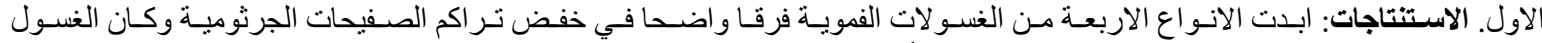

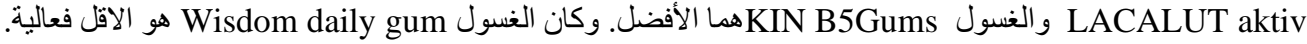

\begin{abstract}
Aims: Study and compare the effectiveness of different mouthwashes on dental plaque and assess the efficiency of text messages on the response of participants. Materials and Methods: The study was approved by Research Ethics Committee board (University of Mosul, College of Dentistry, REC reference No. POP/R.10/1/21). The Participants were (88) dental students, aged between 18 to 25 years (44 males and 44 females). Students with mild to moderate gingivitis were randomly divided into four groups, 22Participants for each. In Group A, participants were advised kin gingival mouthwash, Group B participants used Wisdom daily gum health mouthwash, Group C participants were given LACALUT aktiv, and KIN B5Gums was given to Group D. The subjects were advised to use $10 \mathrm{ml}$ of mouthwash for 30 seconds, twice a day, not immediately after tooth brushing (at least5 minutes after tooth brushing), for 14days. Half of the students in all groups (44students) were motivated on regular intervals by personal text messages, to use mouthwash on regular basis. The parameter was recorded for Plaque index (TQHPI) at day 0 and14. Results: The reduction in dental plaque is highly significant on day 14(T1) compared to baseline values (T0). There are highly significant differences among the four types of mouthwashes used on the reduction of dental plaque. Mouthwash in Group C and D were significantly more effective than Group A and B. Mouthwash in Group A was significantly more effective than mouthwash in Group B. Group C mouthwash did not differ significantly from Group D nor Group A mouthwash in plaque build-up reduction. Conclusions: The four mouthwashes used has good efficacy in reducing dental plaque and there were differences in efficiency between them. KIN B5Gums and LACALUT aktiv mouthwashes were the most effective. Wisdom daily gum mouthwash was the least effective.
\end{abstract}

Keywords: Mouthwashes, Text Messages, Plaque, Dental Students

Fadhil RA., Gasgoos SS. Effect of Different Mouthwashes and Text Messages on Plaque Accumulation for Dental Students. Al-Rafidain Dent J. 2021; 21(2):260-269.

DOI: 10.33899/rden.2021.129084.1070Ｏ2020, College of Dentistry, University of Mosul.

Received: 10/12/2020 Sent to Referees: 15/12/2020 Accepted for Publication: 10/1/2021

This is an open access article under the CC BY 4.0 license (http://creativecommons.org/licenses/by/4.0/ 


\section{INTRODUCTION}

The mouth is the mirror of the body and therefore the health of the mouth has been closely related to systemic health. Periodontal disease is one of the most frequent oral diseases in the world ${ }^{(1)}$.Gingivitis, the mildest sort of periodontal disease, is caused by the dental plaque that accumulates on teeth nearby the gingiva ${ }^{(2)}$. A direct relationship has been verified between dental plaque levels and the severity of gingivitis (3). Although mechanical plaque control may effectively prevent gingivitis if carefully applied, the wide distribution of gingivitis presented in the general population shows that additional measures can prove beneficial. Chemotherapeutic agents have been suggested to be useful adjuncts to the daily oral home care within the control of dental plaque and gingivitis (1). A number of chemical agents are advocated like fluorides, Chlorhexidine, quaternary ammonium compounds (Cetylpyridinium chloride), essential oils, triclosan and sanguinarine, which are either available like a toothpaste/dentifrice or as mouthwash (mouthwash). Among these, chlorhexidine gluconate is considered as the gold standard in dentistry for the prevention of bacterial plaque (3). Mouthwashes containing chlorhexidine are usually available over the counter ${ }^{(4)}$. Chlorhexidine mouthwash is very effective but has certain side effects as brown discoloration in the teeth, bitter taste and oral mucosal erosion
${ }^{(5)}$.Text messages are relatively inexpensive, easily modified, sent directly to individuals, and a part of many individuals' daily life. Many studies utilize text messages as a reminder to help improve health care services ${ }^{(6)}$.

Despite of the popularity of the antimicrobial agents usually found in markets, there is little information about their efficacy on the control of bacterial plaque. Therefore, this study is to gather information about the efficacy of the commonly available antimicrobial agents used to prevent dental plaque accumulation. Furthermore, to help the clinicians to select of the most effective plaque-preventing mouthwash when different products are existing.

\section{MATERIALS AND METHODS}

\section{Study design}

This study was a parallel, singleblinded, randomized comparative clinical study. The study was conducted during the periods 2019-2020 at Dental College of Mosul University.

\section{Subjects and materials used}

The participants, dental students were selected from the age group 18 to 25 years (7) (44 males and 44 females). The students with mild to moderate gingivitis were randomly divided into four groups (22 student in each group) to receive four types of commonly available mouthwashes in Mo- 
sul city, one type for each group. These are as follow:

- Group A: Chlorhexidine Digluconate $0.12 \%$ with $0.05 \%$ (226 ppm) Sodium Fluoride, Aqua, Sorbitol, Glycerin, PEG40 Hydrogenated Castor oil, Allantoin Panthenol, Sodium Methylparaben, Aroma, Citric Acid, Methyl Salicylate, Sodium Saccharin, Cetylpyridinium Chloride, Menthol, Sodium Propylparaben, Eugenol, d-Limonene, Cinnamal (kin gingival mouthwash).

- Group B: Chlorhexidine Digluconate $0.12 \%$ with $0.05 \%$ (225 ppm) Sodium Fluoride, Citric acid, Potassium citrate, Sodium saccharine, Aqua, Glycerine, Sorbitol, PEG-40 hydrogenated Caster oil, Aroma (Wisdom daily gum health mouthwash).

- Group C: Chlorhexidine Digluconate $0.2 \%$ with aluminum fluoride of $225 \mathrm{ppm}$ (Olaflur), Potassium Acesulfame, Aqua, Glycerin, PEG-40 Hydrogenated Castor oil, Propylene Glycol, Aroma, Zinc sulfate, Aluminum lactate (LACALUT aktiv mouthwash).

- Group D: Cetylpyridinium chloride (CPC) $0.05 \%$, provitamin B5 and vitamin B3, zinc lactate, Xylitol $1.00 \%$, Sodium fluoride $226 \mathrm{ppm}(0.05 \%)$ mouthwash (GingiKIN B5 / KIN B5 Gums Mouthwash).

\section{Inclusion and exclusion criteria}

To be eligible for the study the subsequent inclusion and exclusion criteria were applied:

\section{Inclusion criteria:}

Students with no systemic disease, who gave an informed consent, agreement to delay any elective dental treatment as oral prophylaxis, and agreement to fulfill the study visits was included within the study. The ages of the students are between 18 to 25 years having mild to moderate gingivitis was defined by gingival index score between 0.1-2.0 (by Loe and Silness will record), plaque index of 1 or more (by Turesky-Gilmore-Glickman modification of Quigley Hein plaque index) was included in this study.

\section{Exclusion criteria:}

Uncooperative students, students with severe mal-alignment of teeth, orthodontic appliances, removable partial dentures; students already using mouthwash, tobacco consumers, or having smoke or hookah. Students with any medical or pharmacological history that may compromise the conduct of the study were excluded.

\section{Sample size and randomization}

The sample size was defined in 22 students for each group (11males and $11 \mathrm{fe}$ males) to possess a two-sided five percent (5\%) significance level and a power of eighty percent $(80 \%)$, so given an anticipated dropout rate of $10 \%{ }^{(7)}$. Sample size was involved total 88 students, half of them are males and others half are females. Randomization; a computergenerated list of random selected numbers was used to allocate the participants into four groups of 22 participants each. The 
randomization sequence was be created using SPSS Statistic Software 18.0.

\section{Instrument and Equipments}

1. Disposable Gloves and masks.

2. Dental Mirrors.

3. Periodontal probes (WHO probes).

4. Kidney dishes.

5. MGS Disclosing tablets.

6. Disposable cups and towels.

7. Dental chair with suitable light.

\section{Methods}

\section{Dental/ Clinical examination}

Clinical examination was carried out for each student under standardized conditions. Students were examined using plane mirrors and WHO periodontal probes, sitting on dental chairs. A special form was recorded firstly before clinical examination includes general information and some questioners to standardize the sample. Interventions; All students signed informal consent and undergone to the same treatment. The same operator was performing all treatments and all outcome measurements.

\section{Plaque index}

Plaque was scored using TureskyGilmore-Glickman modification of Quigley-Hein plaque (TQHPI) ${ }^{(8)}$, With the TQHPI, facial and lingual non restored surfaces of all the teeth except third molars were scored after using disclosing agents, and The Scoring was as follows:

\begin{tabular}{|c|c|}
\hline Scores & Criteria \\
\hline 0 & No plaque \\
\hline 1 & Separate flecks of plaque at the cervical margin of the tooth \\
\hline 2 & $\begin{array}{l}\text { A thin continuous band of plaque (up to one } \mathrm{mm} \text { ) at the cervical mar- } \\
\text { gin of the tooth }\end{array}$ \\
\hline 3 & $\begin{array}{l}\text { A band of plaque wider than one mm but covering less than one-third } \\
\text { of the crown of the tooth }\end{array}$ \\
\hline 4 & $\begin{array}{l}\text { Plaque covering at least one-third but less than two-thirds of the } \\
\text { crown of the tooth }\end{array}$ \\
\hline 5 & Plaque covering two-thirds or more of the crown of the tooth \\
\hline
\end{tabular}

\section{Periodontal clinical examination}

The clinical examination was done in sequence to examine at least 5 to 6 students daily until reaching the required sample size. The Turesky-GilmoreGlickman modification of Quigley-Hein plaque index was recorded at 0 then 14 days and all records were maintained on a record chart. Before the baseline examination, the students were refrained from conducting oral hygiene for at least eight hours, but no more than 18 hours ${ }^{(9)}$ and also from eating, drinking in these period.

First examination (T0) 
At baseline (T0) plaque was be evaluated with Turesky-Gilmore-Glickman modification of Quigley-Hein plaque index. A disclosing agent containing erythrosine and food green was used to disclose plaque before recording; MGS Disclosing tablets was be used (as seen in the Figure). Each student has received motivation; oral hygiene and mouthwash usage instructions were given. All mouth- washes were over-wrapped to maintain the blinded study design. All the students were still on their daily regular tooth brushing using standardize toothbrush and toothpaste with modified bass method brushing technique. The instructions to use the mouthwash to be explained and prescribed in the same way to each participant, as follows:

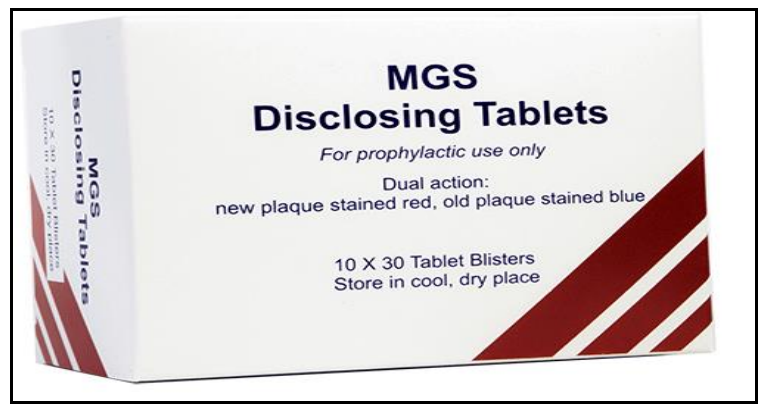

Figure (1) : MGS disclosing tablets

Oral rinse (vigorous swishing in mouth) with $10 \mathrm{ml}$ of given mouthwash for 30 seconds, twice a day (in the morning and in the evening), not immediately after tooth brushing, according to manufacturer instructions, for 14 days $^{(7)}$. Students must also wait until all traces of toothpaste are removed before rinsing with Chlorhexidine. And best utilized time of mouthwash is at least five minutes after the tooth brushing. A measuring cup was given for the each patient to measure the quantity of the mouthwash to use. They were asked not to eat or drink

anything for half-an-hour after using the rinse.
Half of students in each group (11 students) were motivated on regular intervals by personal text messages, to use mouthwash on regular basis. Text messages were done twice a day, after breakfast and after dinner to remind and ensure the usage. These text messages were made every day to half of the students in each group (11 students).

\section{Final examination (T1)}

Compliance was checked with the help of a reminder sheet to be filled by the students daily after using the mouthwash to note down the time at which they rinse on. These compliance sheets were checked by the investigator during subsequent examinations (T1). At the end of the study, the participants were asked to return their 
(empty or not) bottles of mouthwashes to measure the remaining fluid. In the subsequent examinations (T2) the examiner was posed a direct question to participant to check if the mouthwash was used according to instruction for 14 days (if not participant was be excluded from study). In the day 14 (T1), all the mentioned clinical parameters were be re-assessed by same examiner under standard dental office and light source.

\section{RESULTS}

Preliminarily, the KolmogorovSmirnov test used to examine data distribution; it confirmed that data is normally distributed. Changes from baseline (T0) to different time interval (T1) in TQHPI plaque index was analyzed by paired t-test (Intragroup). The paired t-test was executed independently for each mouthwash, to analyze the data pre and post-treatment. Variables represented the variations between observation times (T0, T1). The results were considered statistically significant if they occurred with probability less than or equal to $0.05(p \leq 0.05)$.

The paired t-test result showed that there are significant differences (decrease) between pre and post treatment where the differences of mean TQHPI Plaque index between 0 to 14 th day were $(1.8733$ $\pm .7061,2.1619 \pm .7270,1.3408 \pm .5352$ and $1.6631 \pm .3601)$ respectively in groups $\mathrm{A}$, $\mathrm{B}, \mathrm{C}$ and $\mathrm{D}$ as seen in Tables (1).

Table (1): TQHPI Plaque index Paired t test Statistical results

\section{Paired Differences}

\begin{tabular}{|c|c|c|c|c|c|c|c|c|c|}
\hline \multicolumn{2}{|c|}{ Mouthwashes groups } & \multirow[t]{2}{*}{ Mean } & \multirow[t]{2}{*}{$\begin{array}{l}\text { Std. De- } \\
\text { viation }\end{array}$} & \multirow[t]{2}{*}{$\begin{array}{l}\text { Std. Er- } \\
\text { ror Mean }\end{array}$} & \multicolumn{2}{|c|}{$\begin{array}{l}\text { 95\% Confidence In- } \\
\text { terval of the Differ- } \\
\text { ence }\end{array}$} & \multirow[t]{2}{*}{$\mathbf{T}$} & \multirow[t]{2}{*}{ df } & \multirow[t]{2}{*}{$\begin{array}{l}\text { Sig. (2- } \\
\text { tailed) }\end{array}$} \\
\hline & & & & & Lower & Upper & & & \\
\hline & & & & & 1.5603107 & 2.1864529 & 12.4 & 21 & \\
\hline & & 16 & & & 1.8 & & & & \\
\hline Pair 3 & & 1.3408182 & .53 & & 1.1035054 & 1.5781309 & 11.750 & 21 & $.000 * *$ \\
\hline iir & D1 - D2 & 1.6631682 & .3601566 & .0767856 & 1.5034837 & 1.8228527 & 21.660 & 21 & $.000 * *$ \\
\hline
\end{tabular}

Df: Degree of Freedom, ${ }^{* * H i g h l y ~ S t a t i s t i c a l l y ~ S i g n i f i c a n t ~ D i f f e r e n c e ~ a t ~} p \leq 0.01$ For Each Group of mouthwashes.

The ANOVA test was used to check if there are statistically significant differences among the four mouthwashes groups. Duncan's post hoc was applied for the multiple comparisons. ANOVA analysis for TQHPI Plaque index data (Table 2) and the Duncan's post hoc multiple comparisons (Table 3) illustrated the statisti- cally significant differences among the four groups of mouthwashes. Mouthwashes in Group D and Group C were significantly more effective than mouthwashes in Group $\mathrm{A}$ and $\mathrm{B}$ in reducing plaque formation. Mouthwash in group A was significantly more effective than mouthwash in Group B. Group C mouthwash not differ 
significantly from group D mouthwash nor group A mouthwash in plaque build-up reduction.

Statistical analysis (Independent t-test) was done; between the Students who received text messages (44) to remember them to use mouthwashes and other who did not receive (44); to show if there is a significant difference between them. The Statistical test was analyzed post treatment scores for plaque Turesky-GilmoreGlickman modification of Quigley-Hein plaque index (TQHPI).

Table (4): illustrates that there is no significant difference on plaque reduction between two groups.

Table (2): TQHPI Plaque index ANOVA statistical result

\begin{tabular}{lccccc}
\hline \multicolumn{1}{c}{ ANOVA } & $\begin{array}{c}\text { Sum of } \\
\text { Squares }\end{array}$ & Df & Mean Square & F & Sig. \\
\hline Between Groups & 6.226 & 3 & 2.075 & 7.594 & $\mathbf{. 0 0 0}^{* *}$ \\
Within Groups & 22.959 & 84 & .273 & & \\
Total & 29.185 & 87 & & & \\
\hline
\end{tabular}

Df: Degree of Freedom , ${ }^{* *}$ Highly Statistically Significant Difference at $p \leq 0.01$ For Each Group of mouthwashes.

Table (3): Duncan's post hoc multiple comparisons

\begin{tabular}{cccc}
\hline $\begin{array}{c}\text { Mouthwashes } \\
\text { groups }\end{array}$ & Mean & N & Std. Deviation \\
\hline $\mathbf{A}$ & $1.853673 \mathrm{~B}$ & 22 & .4346231 \\
$\mathbf{B}$ & $1.483727 \mathrm{C}$ & 22 & .5266134 \\
$\mathbf{C}$ & $2.061550 \mathrm{AB}$ & 22 & .6108977 \\
$\mathbf{D}$ & $2.185568 \mathrm{~A}$ & 22 & .5038342 \\
Total & 1.896130 & 88 & .5791880 \\
\hline * Different Letters Indicate Statistically Significant Difference Within the Same Column (Vertically) \\
at $\mathrm{p} \leq 0.05$.
\end{tabular}

Table (4): TQHPI Plaque index independent $t$-test Statistical results

\begin{tabular}{ccccccc}
\hline $\begin{array}{c}\text { Text message } \\
\text { groups }\end{array}$ & N & Mean & T value & Sig & Std. Deviation & Std. Error Mean \\
\hline Group 1 & 44 & 1.950111 & .873 & .385 & .5436387 & .0819566 \\
Group 2 & 44 & 1.842148 & & & .6141761 & .0925905 \\
\hline
\end{tabular}

\section{DISCUSSION}

The primary outcome observed in this study is the reduction in the dental plaque after use of the mouthwashes. The reduction in the dental plaque is highly signifi- cant on day 14 (T1) compared to baseline values $(\mathrm{T} 0)$. There are highly significant differences between the four types of mouthwashes used on reduction of the dental plaque. 
Plaque index (TQHPI) showed high significant reduction between the first (T0) and second (T1) visits in all study groups. This may indicate the good oral hygiene compliance of the participants as well as good maintaining of oral hygiene over the time period of this study, and also may related to the chlorhexidine antimicrobial action (in group A, B and C) that has antimicrobial effects against Gram-positive, Gram-negative, fungi and yeast ${ }^{(10)}$. A study carried out by Van Strydonck et al. (11), showed that Chlorhexidine mouthwash, as an addition to the mechanical oral hygiene, provides significant decreases in the dental plaque formation similar to the result of this study. And also may related to the Cetylpyridinium chloride (CPC) antimicrobial action (group D) The result obtained in this study is in agreement with other studies reported in the systematic review carried out by Haps et al. ${ }^{(12)}$ concluded that CPC containing mouthwashes, when used as adjuncts to oral hygiene (tooth brushing), provide significant additional benefit in reducing dental plaque accumulation.

The results of this study prove that the four mouthwashes have variable antiplaque activity depending on their major active chemical components. Where the group D mouthwash (CPC 0.05\%) and group $\mathrm{C}$ mouthwash (CHX 0.02\%) appeared to provide dental plaque similar inhibitory action, and exhibited the best activity on plaque reduction comparing to group A and B. whereas overall group B mouthwash (CHX 0.01\%) was less effective.

Based on this study the mouthwashes containing CPC 0.05\% (group D mouthwash) or CHX $0.2 \%$ (group C mouthwash) exhibited best activity on plaque reduction; both provide similar plaque inhibitory action this may be due to the antiplaque property of CHX and CPC and concentration of $\mathrm{CHX}$ used in mouthwash $\mathrm{C}$ was $0.2 \%$ and routine oral hygiene methods were also advised. Mouthwash in group A was significantly more effective than mouthwash in Group B this result is in agreement with Zarandi ${ }^{(13)}$ which reveals that Kin Gingival (group A) mouthwash has a greater effect than the chlorhexidine on clinical parameter and is recommended to be used for dental plaque chemical inhibition. Group C mouthwash did not differ significantly from group D mouthwash nor group A mouthwash in plaque build-up reduction where all of them effective antibabacterial and antiplaque substances. Mouthwash in group A was significantly more effective than mouthwash in Group $\mathrm{B}$ this result is agreement with Zarandi ${ }^{(13)}$.

Text messages, In this study all the participants (88) was divided always 2 groups the first group (44) did not receive the text messages and the second group (44) received the text messages to remember them to use the mouthwashes, Independent t-test was done to show if there a significant differences between the two groups on plaque reduction. 
The Statistical test was analyzed post treatment scores for plaque; TureskyGilmore-Glickman modification of Quigley-Hein plaque index (TQHPI), and the result revealed no significant differences on plaque reduction between two groups as showed in Table (4). This may be due to confirmed oral hygiene instructions given to all participants to utilize the mouthwashes twice daily and also may be due to that the participants are dental students who are educated and awareness of adherence to treatments. And also repeated daily messages may be tolerated by participants so that no significant differences present between the two study groups.

\section{Conclusions}

In this study, all four mouthrinses used had good clinical efficacy in reducing dental plaque when utilized alongside tooth brushing with toothpaste. Mouthwashes lacalut Activ and KIN B5 appeared to provide similar plaque inhibitory action. lacalut Activ mouthwash did not differ significantly from KIN B5 mouthwash nor kin gingival mouthwash in plaque build-up reduction. Wisdom daily gum mouthwash was the least effective in reducing dental plaque among the four mouthwashes used in this study. This study showed no difference between text message and without text message groups regarding plaque reductions.

\section{REFERENCES}

1. Moran J., Pal D., Newcombe R., and Addy M. Comparison of phenolic and $0.2 \%$ chlorhexidne Mouthwash on the develop- ment of plaque and gingivitis. Clin Prev Dent 1991; 13(4):31-35.

2. Pihlstrom B., Michalowicz, B., and Johnson, N. Periodontal diseases. the lancet 2005; 366(9499): 1809-1820.

3. Fine HD. Chemical agents to prevent and regulate plaque development. Perio 2000 1995; 8:87-107.

4. James P, Worthington HV, Parnell C, Harding M, Lamont $\mathrm{T}$, Cheung A, Whelton H,and Riley P. Chlorhexidine mouthwash as an adjunctive treatment for gingival health. Cochrane Database of Systematic Reviews 2017; 3. DOI:10.1002/14651858.CD008676.

5. Van der Ouder FJG. Anti-plaque agents. Rationale and prospects for prevention of gingivitis and periodontal diseases. J. Clin. Periodon. 1991; 18(6): 447-54.

6. Rohman, L., Maruswezki, D., Cam, N.B. The impact of a text messaging service on orthopaedic clinic did not attend rates. $J$. Telemed. Telecare, 2015; 21 (7), 408-413.

7. Guerraa F., Pasqualottoa D., Rinaldoa F., Mazura M., Corridorea D., Nofronib I., Ottolenghi L. and Nardi G.M. Therapeutic efficacy of chlorhexidine-based mouthwashes and its adverse events: performance-related evaluation of mouthwashes added with anti-discoloration system and cetylpyridinium chloride. Int J Dent $\mathrm{Hy}$ giene. 2019; 17: 229-236.

8. Turesky S, Gilmore ND and Glickman I. Reduced plaque formation by the chloromethyl analogue of victamine C. J Periodontol 1970;41:41-43. 
9. Sharma N, Charles CH, Lynch MC. Adjunctive benefit of an essential oil-containing mouthwash in reducing plaque and gingivitis in students who brush and floss regularly: a sixmonth study. J Am Dent Assoc 2004; 135(4): 496-504

10. Nairn Wilson, Rakhee Patel, Jennifer Gallagher and Iain Chapple Question from practice: How to select the right mouthwash. The Pharmaceutical Journal 2014; 292 (7795): 119.

11. Van Strydonck DAC, Slot DE, Van der Velden U and Van der Weijden F. Effect of a chlorhexidine mouthwash on plaque, gingival inflammation and staining in gingivitis patients: a systematic review. J Clin Periodontol 2012; 39: 1042-1055.
12. Haps S, Slot DE, Berchier CE, van der Weijden GA. The effect of cetylpyridinium chloride-containing mouth rinses as adjuncts to toothbrushing on plaque and parameters of gingival inflammation: a systematic review. Int J Dent Hyg 2008; 6:290-303.

13. Zarandi Ali Comparison of clinical effects of kin gingival mouthwash with chlorhexidine $0.2 \%$ mouthwash in generelized mild to moderate chronic Periodontitis. Thesis. $\mathrm{Ta}$ briz university of medical sciences, college of dentistry; 2018 Tabriz, Iran 